\title{
LA-5640-MS
}

Informal Report

UC-20

Reporting Date: May 1974 Issued: June 1974

\section{On CTR Breakeven Including Energy Transfer and Recovery Efficiencies}

by

D. A. Freiwald

R. A. Gerwin 
This report was prepared as an account of work sponsored by the United States Government. Neither the United States nur the United States Atomic Energy Commission, nor any of their employees, nor any of their contractors, aubcontractors, or their emplovees, makes any warrenty, express or im. phiod, or asumes any logl liability or responsibility for the accuracy, completeness or usefulness of any information, apparatus, product or process disclosed, or represents that its use would not infringe privately owned rights.

In the interest of prompt distribution, this LAMS report was not edited by the Technical information staff.

Printed in the Uniled States of America. Available from National Technical Information Service

U.S. Department of Commerce

5285 Port Royal Hoad

Springtield, Virginia 22151

Price: Printed Copy \$4.00 Microliche $\$ 1.45$ 
ON CTR BREAKEVEN INCLUDIHG ENERGI TRAHSFER AHD RECOUERY EFEICIENCIES

by

D. A. Freiwald (L-5) and R. A. Gerwin (CTR-6)

\begin{abstract}
Breakeven conditions for controlled sherwonuclear reactions (CTR) are addressed. Stuple but general equations are presented thich include energy transfer $\left(\varepsilon_{1}\right)$ and recovery $\left(\eta_{1}\right)$ efficlencles for energies in varlous forms. A general equation for breakeven is develoged, showing the sensitivity of the efficiency factors. Each experiment will have lis ow breakeven curve, depending on the $\eta$ 's and $\varepsilon$ 's.
\end{abstract}

\section{INTRODUCTION}

The simple Lawson cricerion for CTR breakeven 1,2 dees not include separate transfez and zecovery efficiencies for each of various energy forns. We actempt here to updace the breakeven criterion by formulating breakeven in general but still sotethat simple terns amenable to easy use. We include transfer efficiencies, i.e., that fraction $\varepsilon_{1}$ of input energy $E_{0}$ that ends up in sose other fore (e.8., thermal, radiation, fields). We also include recovery efficiencies $n_{1}$, l.e., the fraction of energy In the system in the $1^{\text {th }}$ form that can be recovered in one manner or another for recycling or conversion.

The efficiencies can be invoked into general equations together with only a few assumptions, to give solutions for $n \tau=f\left(T, \eta_{i}, \epsilon_{1}\right)$ where $n$ is the Ion numer density, $T$ is the plasm confinement time, and $I$ is the plasma temperature. A point that emerges is that each expericent has its own breakeven curve, depending on the $E_{1}$ 's and $n_{1}$ 's.

\section{GENERAL EQUATIONS}

Consider an equal wixture of deuterium (D) and tritium (T) in a pulsed CTR experiment that is powered with some input energy $E_{0}$ needed for the first cycle. This energy will appear in various forms in the system.

We assume 3 Maxwellian distribution in the plasma, nonrelativistic species, and $T=T_{e}=T_{1}$. Then for inpul energy balance, the terms of prime but stili general interest are:

$$
E_{0}=E_{p}+E_{b}+E_{k}+E_{c}+E_{t}=E_{0} E_{1} E_{1}
$$

where,

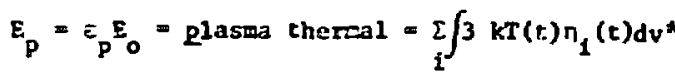

$$
\begin{aligned}
& E_{b}=\varepsilon_{b} E_{0}=\text { Bressstrahlung }= \\
& =\iint 5.35 \times 10^{-31} n_{\mathrm{e}}(t) \sum_{1}\left[n_{1} z_{i}{ }^{2}\right] T^{1 / 2} d v d t_{i} \\
& E_{k}=\varepsilon_{k} E_{0}=\text { plasea } \underline{k} \text { lnetic } \\
& -\frac{1}{2} w w^{2}=\frac{1}{2} \sum_{1} a_{1} n_{1} \vec{w}^{2}
\end{aligned}
$$

$E_{c}-\varepsilon_{c} E_{0}=$ cyclotion (with pressure balance)

$$
\begin{array}{r}
=\iint_{v} 5 \times 10^{-32} \mathrm{n}_{\mathrm{e}}{ }^{2} \mathrm{~T}^{2} d v d t ; \\
E_{D}=\varepsilon_{D^{2}} E_{0}=
\end{array}
$$

energy in electric and/or magnetic fields,

and $\mathrm{d} v=$ elemental volume, $v_{\mathrm{p}}, v_{\mathrm{s}}$, and $\mathrm{v}_{\mathrm{kE}}$ are, respectively, the volumes assoctated with the thermal plasma, the skin depth, and the plasma in global motion, with dt = time increment, $Z$ = stripping number, $n_{1}=$ species number density, $a_{1}=$ mass of $1^{\text {th }}$ species particle, and $0 \leq \varepsilon_{1}<1, \sum_{1} \varepsilon_{1}=1$.

*Including electrons but neglecting binding energies. 
In Eqs. (1) we have, in a sense, used a peculiar energy breakdown by distributing $E_{0}$ betreen $E_{p}, E_{b}$ and $E_{c}$ independently when they are in fact coupled, both together and with $E_{w^{*}}$. For exarple, $E_{b}$ and $E_{c}$ alght be drawing upon $\mathrm{E}_{\mathrm{p}}$ during the confinement time. Hevertheless, at the end of that tioe, one certainly hes energy balance, $E_{0}=E_{p}+E_{b}+E_{c}+\ldots$. , where the RHS of $\mathrm{Eq}$. (1) contains the energy left in the plase at that tine, the Brensatrablung radiation up to that tine, etc. Used above, $E_{p}$ is the plasea therenl energy at the end of confinewent time, and what follows implies the assumption that $E_{p}$ (end) $\sim E_{p}$ (average, during $\tau$ ). For detail, coupled rate equations should also be considered, ${ }^{3}$ which in general would Include bootstrap beating from fusion products. ${ }^{4}$ Wonecheleas, we proceed here with the format of Eqs. (1) because it is sinflar to Lawson's; ${ }^{1}$ and gives results that are instructive in terms of functional dependencies and sensitivities.

We now assue that impurities are negligfble, and $n=\varepsilon_{1} n_{1}\left(n_{0}=n_{T}=1 / 2 n, n_{d} n_{T}=1 / 4 n^{2}\right)$. whence

$$
P_{b}=5.35 \times 10^{-31} \mathrm{a}^{2} \mathrm{~T}^{1 / 2},
$$

and

$$
P_{c}=5.0 \times 10^{-32} n^{2} T^{2}
$$

We further assume that wean values can be assigned to $T$, the various $V^{\prime} s, t^{\prime} s$, and $n$. This then allows the Introductios of notanlization factors:

$$
\begin{aligned}
& \xi_{c, b, k}=v_{c, b, k} / v_{p}, \\
& \phi_{c, b, k}=t_{c, b, k} / t_{p} .
\end{aligned}
$$

where $t_{p}=\tau$, and $v_{p}=v$ (volume).

Thus Eq. (1) my be rewritten as

$$
E_{0}=E_{0} \sum_{1} \varepsilon_{1}
$$

$3 n k T+\phi_{b} E_{b} P_{b} V \tau+\phi_{c} \xi_{c} P_{c} V \tau+\phi_{k} E_{k} \lambda_{n} V+E_{b}$,

where $\lambda$ tnvolves the particle asses and wean velocity of the total wass. Using $E_{0}=\operatorname{nkTV} / \varepsilon_{1}$, and solving Eq. (6) for E: yields:

$$
\begin{gathered}
E_{D} \cdot 3 n k T V\left(\frac{1}{\varepsilon_{1}}-1\right) \\
-\phi_{b} \xi_{b} P_{b} V \tau-\phi_{c} \xi_{c} P_{c} V \tau-\phi_{k} \xi_{k} \lambda n v .
\end{gathered}
$$

We now consider breakeven. Note for DT:

$$
\mathrm{D}+\mathrm{T}+\mathrm{He}^{(4)}(3.5 \mathrm{MeV})+\mathrm{n}(14.1 \mathrm{MeV})
$$

In discussing breakeven it was assumed by Lawson that the neutron energy was lost, and thus

$$
\begin{aligned}
& P_{f}=\text { fusion poser density }= \\
& =5.6 \times 10^{-13}<0 \%>n_{d} n^{\prime}
\end{aligned}
$$

where

$$
\left\langle\sigma v>\left.\right|_{\mathrm{DT}}-\frac{3.68 \times 10^{-12}}{\mathrm{~T}^{2 / 3}} \exp \left(-\frac{19.94}{\mathrm{~T}^{1 / 3}}\right),\right.
$$

for lower T'g (see page 18, Ref. 2 ). Here we generalsze and Include neutron energy, by intrcducing the quantity $f \equiv E_{I}+f_{W}$, where $f_{I}$ relates to the ion energy and $f_{k}$ relaces to the neutron energy such that $0 \leq f \leq 5,0 \leq f_{I} \leq 1$, and $0 \leq f_{K} \leq 4$. The $f^{\prime} s$ are caken to be the fractions of recoverable fusion energy.

We nou define various $\eta^{\prime}$ 's to be recovery efficiencies. A stoflar spproach is used by Nozawa and Stelnex. 5 Then for breakeven.

$$
\left(n_{I} E_{I}+n_{H F} f_{F}\right) P_{F} \nabla T+E_{0} \sum n_{I} \varepsilon_{I}=E_{0}
$$

Introducing $P_{f}=n^{2} F$ where $F=\langle\sigma v\rangle, P_{b}=a^{2} B$, and $P_{c}=a^{2} c$, then Eq. (11) aay be remritten as:

$$
\begin{aligned}
& \left(n_{I} f_{I}+n_{w} F_{A}\right) a^{2} F V \tau+n_{p} 3 n k T V+n_{b} \phi_{b} E_{b} n^{2} B V \tau \\
& +n_{k} \phi_{k} \xi_{k} \lambda \pi \nu+n_{c} \phi_{c} \xi_{c} n^{2} c V \tau+n_{D} E_{D}= \\
& 3 n k \tau V+\phi_{b} \xi_{b} n^{2} a V \tau+\phi_{k} \xi_{k} \lambda n V \\
& +\phi_{c} \xi_{c} n^{2} c v \tau+E_{w}
\end{aligned}
$$

where $F, B$, and $C$ are only functions of $T$.

Substituting Eq. (7) into Eq. (12) and rearranging.

$$
\left.n T\right|_{\text {REQ'D }}=
$$

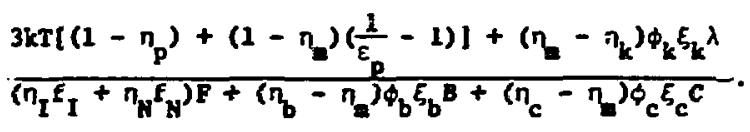

If global kinetic energy and cyclotron radiation are negligible $(\lambda=6=0)$, Fq. (13) becomes: 


$$
\begin{aligned}
& \left.n \tau\right|_{\text {SEQ'D }}= \\
& \frac{3 k T\left[\left(1-\eta_{p}\right)+\left(1-\eta_{p}\right)\left(\frac{1}{\varepsilon_{p}}-1\right)\right]}{\left(\eta_{I} \tilde{F}_{I}+\eta_{w} f_{N}\right) F+\left(\eta_{b}-\eta_{D}\right) \phi_{b} \xi_{b} B},
\end{aligned}
$$

which shows, as in Eq. (13), that if $\eta_{n}=1$ then $n \tau$ beqcomes independent of $\varepsilon_{p}$ explicitiy.

$$
\text { If } \varepsilon_{\mathrm{m}}=\eta_{\mathrm{N}}=0, \mathrm{f}_{I}=\phi_{\mathrm{b}}=\xi_{\mathrm{b}}=\varepsilon_{\mathrm{p}}=1, \eta_{\mathrm{p}}=\eta_{\mathrm{b}}
$$

$=n_{I}=1 / 3$, then $E q$. (14) reduces to

$$
\mathbf{n} \tau=\frac{6 \mathrm{kT}}{\mathrm{F}-\mathrm{B}}
$$

which is the classical Lasson criterion. Note that $E_{\text {ti }}=0$ and $E_{p} \sim 1$ are not realistic.

In a real world CTR reactor, kinetic, thermal, cyclotron, bremsstrahlung, and fusion-ion energies will probably be recovered by the same process, with some efficiency $\eta_{i}\left(=n_{p}=n_{b}=n_{k}=n_{c}=n_{I}\right)$, where $n-0.10$ for a modern boiler-steam generator cycle.

With magnetic energy transfer and storage, $\eta_{n}$ may be as high as $\sim 0.62$. (Ref. 6)

Renarks on Rqs. (13-14):

- Because $\eta_{m}$ will probatly exceed $\eta_{k}$, it is desirable tc have $\phi_{k} \xi_{k} \lambda=0$.

- If $\eta_{w}>\eta_{c}, \eta_{b}$, the denominator gets spaller and thus $n \tau$ gets larger, unless $\phi_{b} \xi_{b} B$ and $\phi_{c} \xi_{c} c$ are small. Because $\phi_{b} \xi_{b} B$ cannot be ellminated, it is at least desirable to eliminate $\phi_{c} \xi_{c} c$. In another sense, this 1mplies that a high- $\beta$ plasma is desirable.

- It is desfrable to make $\eta_{N}$ as large as possible.

- It is desirable to make $\eta_{p, b, k, c, I}$ as large as possible.

- If $\eta_{N}$ also is $-0.40, \eta_{p, b, k, c, I}-0.40$, and $n_{\mathrm{g}}-0.62$, then $E_{q}$. (14) becomes

$$
n t=\frac{3 k T\left[0.60+0.38\left(\frac{1}{E_{p}}-1\right)\right]}{\left[0.46 F-0.22 \oint_{b} F_{b} B\right.} .
$$

- It is obviously desirable to make $\varepsilon_{p}$ as large as possible.

- Assuning $\phi_{b}=\delta_{b} \cdot 1$, then Eq. (14b) becomes

$$
r T=\frac{3 k T\left[0.60+0.38\left(\frac{1}{\varepsilon}-1\right)\right]}{\left[0.4 f F-0.22\left(5.35 \times 10^{-31}\right) T^{1 / 2}\right]}
$$

where $F=\langle u v\rangle$.

\section{III.COYPUTATIONAL RESULTS}

For a computational example we use Eq. (14c). We assune that all fuston neutrons and ions can be captured, and thetr energy converted to heat. Thus, $f=5,\left(f_{I}=1, f_{n}=4\right)$.

For computational purposes, Eq. (14c) reads

$$
\frac{3 \mathrm{kT}\left[0.60+0.38\left(\frac{1}{\varepsilon_{\mathrm{p}}}-1\right)\right] 20^{19}}{0.4(5)<\sigma v\rangle-0.22\left(5.35 \times 10^{-31}\right) \mathrm{T}^{1 / 2}} \text {, }
$$

where $k=1.38 \times 10^{-23}\left(\mathrm{~J} /{ }^{\circ} \mathrm{K}\right), T$ is in $\mathrm{keV}$, <OV $>$ is in $\mathrm{cm}^{3}-\mathrm{s}^{-1}$ from page 19 of Ref. 2, and the factor $10^{19}$ in the nucerator converts the numerator to consistent units with the denominator using $1 \mathrm{keV}$ $=10^{7}{ }^{\circ} \mathrm{R}$ and $10^{6} \mathrm{~cm}^{3}=1 \mathrm{~m}^{3}$.

Equation (14d) is plotted in Fig. 1 for various $E_{p}$ and for $T=3$ to $100 \mathrm{keV}$.

Here again it is unrealistic to expect $\varepsilon_{p} \sim 1.0$, 1.e., that all the power supply energy ends up in 3akT. If $\varepsilon_{\mathrm{p}}=10 \%$, Fig. 1 shows that breakeven uould be achieved for $n t=10^{15}$ and $\mathrm{T}>3 \mathrm{keV}$.

For comparison and checks in calculation method consistency, we have computed breakeven on the basis of Lawson's forculation. For computational purposes, Eq. (14a) becomes

$$
\left.\mathrm{nT}\right|_{\text {Lawson }}=\frac{6 \mathrm{kT} \cdot 10^{19}}{\langle 0 \mathrm{v}\rangle-5.35 \times 10^{-31} \mathrm{~T}^{1 / 2}}
$$

with the saze term units as above. Results are shown In Fig. 2, in agreement with Lawson and Linhart's calculations. 7

When the $E_{p}=1.0$ curve of $\mathrm{Yig} .1$ is compared to the Lawson curve show ir. Fig. 2 , it is seen that the former lies further to the left. This is due to

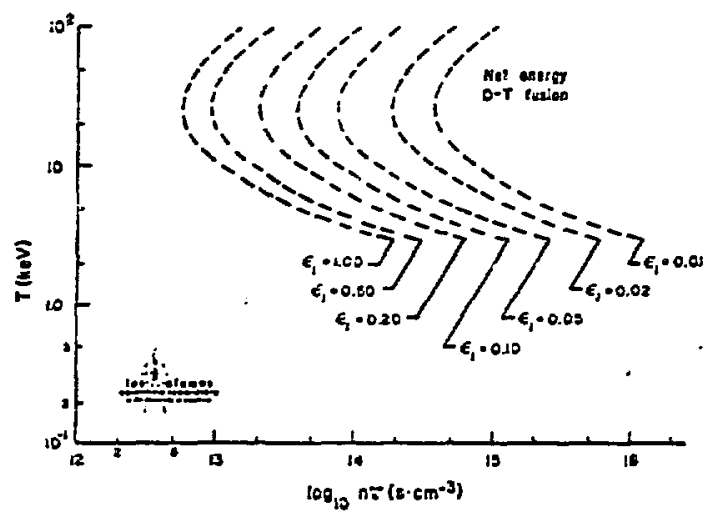

Fig. 1. Breakeven curves for various $\varepsilon_{1}$. 


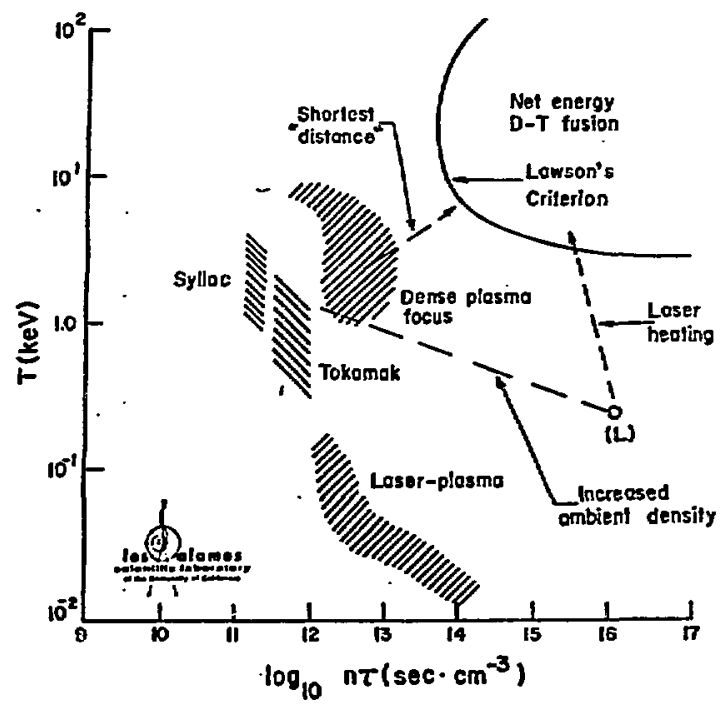

F1g. 2. Lawson's breakeven curve and performance of various plasmi experiments

the fact that we have stipulated that all fusion neutrons are captured, and have used a therma1-to-electric conversion efficiency of $40 \%$, versus Lawson's 337. Because $\sim 80 z$ of the fusion energy is released in neutron kinetic enargy, the $\varepsilon_{p}=0.20$ curve of Fig. 1 should be in rough agreement with the Lawson curve of Fig. 2; Inspection shows that this is the case.

\section{DISCUSSION}

In paet years it was perhaps heuristically valid to compare the status of one's experiment to the Lawson curve (see Fig. 2) because all experiments were a considerable "distance" from breakeven in the $n \tau$ versus $T$ plane. Yet such comparisons are, in general, not valld for at least two reasons:

1) The Lawson criterion assumes $\varepsilon_{m}=0$ and $\varepsilon_{p}-1.0$, which are not realistic.

2) All $\varepsilon_{1}^{\prime} s, \eta_{1}^{\prime} s, \phi_{1}^{\prime} s$, and $\xi_{1}$ 's should at least be considered, via Eq. (13). The point is that each experiment will have its own breakeven

curve, because each experiment has its olm unique $\varepsilon^{\prime} s$ and $n^{\prime} s$.

We have neglected the effects of impurities in the above discussion. High-2 gases containfing wany internal energy states are "soft", 1.e., they cen abscrb energy with relatively less change in temperature as compared to an ideal gas. Unless fully Ionized, the expression for internal energy of a high-z gas will be rather complex. ${ }^{8,9}$ Also, $P_{b} \propto z_{j}^{2}$ [see Eq. (1b)]. Impurities in the plasma should move the breakeven curves (of Fig, 1) upward and to the right.

If $\eta_{\square}$ can be mare greater than the value 0.62 used above, then nt could be reduced. Also note that $f_{N}$ effectively exceed the value of 4.0 used. The energy released by moderated neutrons captured in ${ }^{6}$ Li (tritium breeding) via

$$
6_{\mathrm{LI}}+\mathrm{n} \rightarrow{ }^{4} \mathrm{He}+{ }^{3} \mathrm{~T}+4.6 \mathrm{MeV}
$$

supplies additional energy for conversion. The ${ }^{6} \mathrm{L1}$ constitutes -7.5 atomic percent of natural 11thium. ${ }^{2}$ This added energy will also somewhat reduce the required nt.

Note also that there are many possible paths or trajectories in the nT-vs-T plane for moving towards breakeven.

For example, most magnetically controlled thermonuclear reaction (MCTR) experiments currently fall to the left or lower left of breakeven (see F1g. 2), and experimental efforts are to move performance upward and to the right, across the breakeven curve. But the "shortest distance" may not be the easiest or most efficient.

There are several other paths that could be pursued in the nt-vs-T plane. One possibility which has been discussed is the concept of heating "magnetically prepared" plasmas with lasers.

Shatas, et al, ${ }^{10}$ discuss this possibility for the dense plasma focus whereir a $\mathrm{CO}_{2}$ laser would be used; others ${ }^{11}$ have also been interested in pursuing this.

Hertzberg, Vlases and Hoffman ${ }^{12}$ have been investigating laser heating of a linear $\theta$-pinch. We understand that an experiment for laser heating of the Scylla 1-B $\theta$-pinch is under development. ${ }^{13}$

The basic process involves exciting plasma electrons with laser 1ight, whence the (hotter) electrons then heat lons. To heat the electrons, the laser frequency,

$$
\omega_{2}=\frac{2 \pi c}{\lambda_{2}}
$$

should be close to but greater than the electron plasma frequency

$$
\omega_{p e}=5.63 \times 10^{4} \sqrt{\pi_{e}},\left[n_{e} \text { in } \mathrm{cm}^{-3}\right\} \text {. }
$$


Thus if $\lambda_{\ell} \sim 10.6 \mu\left(\mathrm{CO}_{2}\right)$ then the desired $\mathrm{n}_{e} \sim 10^{19}$ $\mathrm{cm}^{-3}$. In general $\lambda_{\ell}-\mathrm{n}_{e}{ }^{-1 / 2}$.

For current regimes of operation, Fig. 2, laser heating would miss the knee of the Lawson curve. We suggest a different path in the $n T-T$ plane for theoretical consideration. The conceptual path is indicated by the dashed line in Fig. 2:

1. Increase the MCTR nt by raising $n$. For a given power supply energy, $T$ will be lowered. In short, try to move existing MCTR experiment performance under the knee of the breakeven curve to point (L).

2. Then heat with a gas laser, moving performance upward across the breakeve' curve. During this phase the confining fleld may have to be increased.

The laser frequency needed w111 be determined by the $n$ achieved at point (L) and by the laser-beaml plasma coupling efficiency desired.

Note that long (> $100 \mathrm{~ns}$ ) laser pulses would probably be acceptable. For such pulse widths, gas laser efficiency ( $E_{\text {beam }} / E_{\text {power supply }}$ ) way be as high as $25 \% .^{12}$

If good laser-beam/plasma coupling efficiencles, say $80 \%$, could be achieved, then the laser-system/ plasma coupling efficiency might be as high as $-20 \%$. This is extremely loportant for breakeven where it is desirable to maximize the energy transfer from power supplies to plagma 3nkT. Most magnetic fusion experiments are currently operating with power supply to plasma 3nkt efficiencies on the order of 12 .

For example, let $\varepsilon_{\mathrm{m}}$ and $\varepsilon_{\ell}$ represent the fraction of energy transferred from $E_{m}$ (RCTR Power Supply) and $E_{\ell}$ (laser power supply) respectively. For a M/L CTR hybrid

$$
\begin{gathered}
3 n k T=\varepsilon_{\mathrm{m}} E_{\mathrm{m}}+\varepsilon_{\ell} E_{\ell} \\
=\varepsilon_{T}\left(E_{\mathrm{m}}+E_{\ell}\right)=\varepsilon_{T} E_{T O T A L}
\end{gathered}
$$

Here, $\varepsilon_{T}$ is our new $\varepsilon_{p}$ that includes the effect of laser heating. Figure 3 shows $\varepsilon_{T}$ versus $E_{\ell} / E_{m}$ for $\varepsilon_{m}=0.01$ and $\varepsilon_{\ell}=0.20$. Higher $\varepsilon_{m}$ and/or $\varepsilon_{\ell}$ will raise the curve, and vice versa. From the figure, 1f $\left(E_{\ell} / E_{t}\right)=1$, then $\varepsilon_{T}-10.5 \%$, which would be more effictent than either MCTR or LCTR alone; laser/pellet fustion is predicted to be $-7 \%$ efficient, whence the curve of Fig. 3 would ultimately fall of to the right if solid targets are also considered.

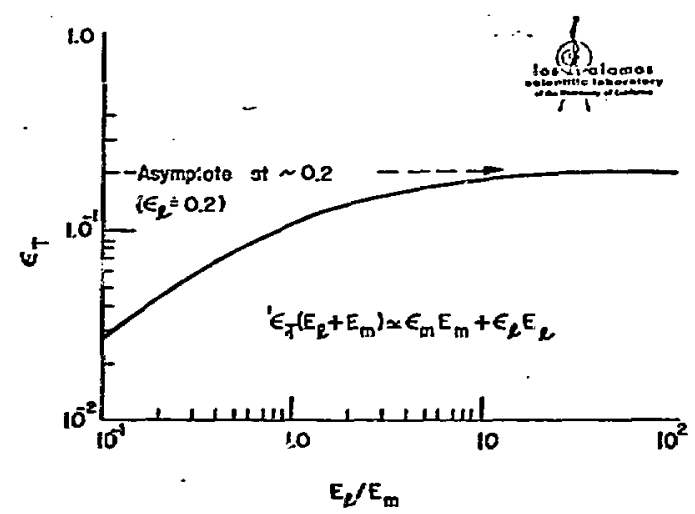

Fig. 3. Total transfer efficlency for various ratios of laser and magnetlc power supply energies.

Other factors such as system $Q$, circulating power, ets., must all be 1nvestigated in a system study to find what point on the $\varepsilon_{T}$ versus $E_{l} / E_{I}$ graph would be optimu for operation. Several scaling laws have already been investigated by Ellis and Saryer, 14,15 though for total laser heating of gaseous DT.

Note that increasing $\mathrm{n}$ (and thus sacrificing $\mathrm{T}$ ) for the cagnetic coopression phase way help reduce certain instability growths. The growth time $\tau_{18}$ $\sim 2 \pi r / v_{e} \sqrt{N}$ where $N$ is the mode number (number of flutes) and $U_{a}=\sqrt{B^{2} / 4 \pi \rho}, B^{2}-P-n k T$. Thus $\tau_{i g}$ $-\sqrt{\rho / B^{2}}-\sqrt{\rho / T}$. Increased $\rho$ and decreased $T$ would both appear to suppress the instability growth; upon laser heating the increased $T$ would tend to be offset by the increased density operation.

The fuston reaction rate $R$ goes as $R-n^{2}\langle C V\rangle$, where, In probable regimes of interest $(e . g .$, point (L) on the $n T-T$ graph), <oV $\propto T^{4}, 1 . e .,\langle\sigma V\rangle$ is very sensitive to $T$ as shown in Fig. 4. With efficlent laser-bead/plaswa coupling, small changes in $T$ can give rapid gains in $\langle\sigma V\rangle$, and thus $R$. The increased $n$ will also ald $R\left(\alpha n^{2}\right)$. The hybrid concept would thus appear to make maximum use of the most sensitive functions. Further theoretical investigation and optimization is needed.

Heating with pulsed relativistic electron beams is another concept being explored, ${ }^{16-20}$ though not particularly in the "dog-leg path" concept shown in Fig. 2. Por electron beat heating, the effect of the excess electrons on bremsstrahlung radiation, and thus on the breakeven equations, needs to be explored. 


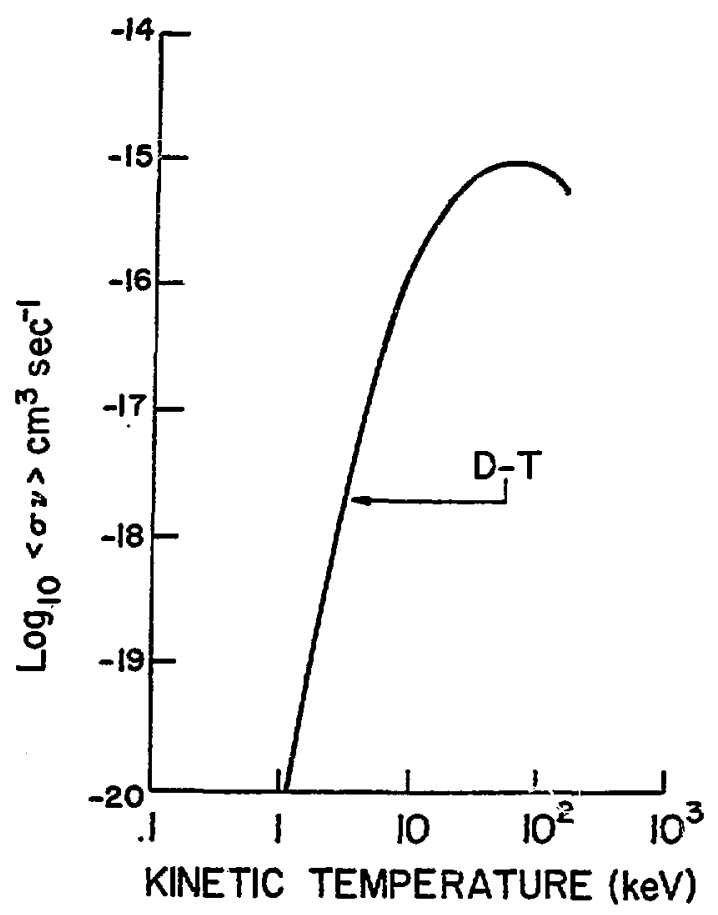

Fig. 4. Maxwe11 velocity-averaged reaction crosssection parameter versus temperature.

The above discussion aiso assumes a Maxwellian plasma. Theory for hybrid experiments might consider the possibility of using an appropriate beam to excite a bump on the tail of the ion distribution function, as is currently under study for so-called two-component Tokamaks.

Obtaining the desired physics is only the first step in developing a CTR concept. The second step Is to achieve sound engineering for a net power CTR reactor in terms of rellabllity, safety, and longevity $^{21}$ (e.g., minimize material damage from radiation). Finally, the third step is to put together a package of the first two steps that will provide economically competitive electrical power. ${ }^{22}$ We suggest that the three steps are distinctly different, and that analysis of all three together via systems studies will suggest most promising directions for pursuit. A system that is relatively simpler and easfer to maintain will be more acceptable to the private power Industry (glven comparative economics) when the time comes for technology transfer.

\section{ACKNOHLEDGEMENTS}

We gratefully acknowledge the heipful discussions with J. Hilliams, J. Mather, K. Ware, and J. Downing. We thank E. Heck for programining the 9820 computer for the various calculations.

We have just learned that the concepts presented in Sections I-III are apparently somewhat similar to those of S. Gralnick of PPPL, ${ }^{23}$ whose work may have been proceeding along parallel lines.

\section{REFERENCES}

1. J. D. Lawson, "Some Criteria for a Power Producing Therwonuclear Reactor," Proc. Phys. Soc, (London) B70, 6 (1957).

2. S. Glasstone and R. H. Loveberg, Controlled Thernonuclear Reactions, D. Van Nostrand (New York, 1960) p. 41.

3. F. Ribe, "Fusion Reactor Systems," Los Alamos Scientific Laboratory Report (in preparation).

4. G. S. Fraley, E. J. Linneour, R. T. Mason, and R. L. Morse, "Thermonuclear Burn Characteristics of Compressed Deuterium-Tritium Micruspheres," Los Alamos Scientific Laboratory Report LA-5403MS (October 1973).

5. M. Nozawa and D. Steiner, "An Assessment of the Power Balance in Fusion Reactors," Oak Ridge National Laboratory Report ORNL-TM-4421 (November 1973).

6. K. I. Thomassen, "Reversible Magnetic Energy Transfer and Storage Systems," Los Alamos Scfentific Laboratory Report LA-5087-MS (1972).

7. J. G. Linhart, "A Note on Several Different Approaches to the Problem of Controlled Fusion," EURATOM-CNEN, Frascati, Italy (1967).

8. J. R. Freeman, "Equilibrium Hydrodynamic Variables Behind Incident and Reflected Shock Waves in Hydrogen," Sandia Laboratories Report SC:-RR-68687, (1968).

9. N. T. Grier, "Equilibrium Hydrodynamic Variables Behind the Primary and Reflected Shock Waves in Argon and Nitrogen," NASA Report TN D-2905 (1965).

10. R. A. Shatas, J. D. Stettler, H. C. Meyer, T. G. Roberts, "Soft X Rays from a Laser-Heated Dense Plasma Focus," J. App1. Phys. 42, 5884 (1971).

11. J. Mather, Los Alamos Scientific Laboratory, Private Communication (Jine 1973).

12. Laser Focus, (April 1973), p. 10.

13. W. El11s and T. Stratton, Los Alamos Scientific Laboratory, Private Communication (April 1974).

14. W. R. Ellis and G. A. Sawyer, Los Alamos Scientific Laboratory Report LA-5434-KS (October 1973).

15. W. R. Ellis, Los Alamos Scientific Laboratory Report LA-5499-MS (February 1974).

16. J. W. Mather, J. P. Carpenter, D. A. Freiwald, K. D. Ware, A. H. Williams, "Electron Beam and Dense Plasma Focus Interaction Experiments," J. App. Phys. 44, 4913 (1973). 
17. D. A. Freiwald, K. R. Prestwrch, G. W. Kuswa, E. H. Beckner, "Neutron Enhancement from Relativistic Electron Beam - Dense Plasma Focus Interactions," Phys. Lett. 36A, 297 (1971).

18. R. A. Gerwin, "Energy loss of a Relativistic Electron Beam in a Plasma," Los Alamos Sctentific Laboratory Report LA-5269-MS (1973).

19. J. Guillory, "Instabilities and Energy Transfer from Relativistic Beam," Fhysics International Company Report PIIR-14-72 (1972).

20. R. N. Sudan, "Applications of Intense Relativistic Electron Beams to Controlled Thermonuclear Reactions," Cornell University Laboratory of Plasma Studies Report LF -130 (July 1973).
21. J. M. WH11 Lams, and T. G. Frank, "Laser Controlled Thermonculear Reactor Materials Requirements," Presented at 1973 Winter Meeting of the ANS (San Francisco, California, November 11-15, 1973).

22. F. T. Finch, E. A. Kern, J. M. Williams, "Laser Fusion Power Plant Systens Studies," USAEC Report CONF-740402 (to be published).

23. S. Gralnick, "Luw Density closed Plasmas," Proc. of CTR Horkshop, Dept. of Nuclear Engineering, University of Arizona, March 18-22, 1974. 